\title{
Sciences et société : nous sommes tous des autodidactes
}

Marie-Pauline Gacoin (marie-pauline.gacoin@synchrotron-soleil.fr)

Responsable de la communication du synchrotron SOLEIL, Présidente de l'association Sciences ACO

Synchrotron SOLEIL, L’Orme des Merisiers, Saint-Aubin, BP 48, 91192 Gif-sur-Yvette Cedex

Chaque jour, lorsque des

particuliers, des industriels,

des classes de collégiens,

de lycéens ou d'étudiants

visitent SOLEIL, les équipes

qui les accueillent relèvent

les mêmes défis : partager

avec eux, en quelques heures,

l'excitation d'un résultat ou

$d^{\prime}$ une manip qui se monte,

aussi complexe ou théorique

soit-elle, attiser leur curiosité,

leur esprit critique et, au-delà

de la surprise visuelle, susciter

leur envie d'en savoir plus.

Pour assumer cette démarche

d'ouverture permanente

aux publics, professionnels et citoyens, certains synchrotrons,

et SOLEIL en particulier,

développent depuis dix ans

une politique volontariste

et des actions gratuites pour

être accessibles à tous

et engager le dialogue sur

la recherche, ses enjeux

et ses résultats.
SOLEIL propose des programmes pédagogiques qui collaborent avec les pratiques scolaires, les offres des musées et des $\operatorname{CCSTI}^{(1)}$, le travail de terrain des associations de culture scientifique et de culture populaire. Le contexte synchrotron est particulièrement propice à cette médiation in situ : pluridisciplinarité, co-activité technologique et scientifique, diversité des métiers, continuum entre la science fondamentale et la science appliquée... L'enjeu est de mobiliser ensemble, jeunes ou moins jeunes, néophytes ou spécialistes, nos connaissances, nos savoirs académiques ou vécus, pour construire aujourd'hui la société de demain.

\section{Partager les connaissances}

Transmettre des connaissances est traditionnellement une activité d'enseignement. Dans un monde désormais numérique qui transporte les centres d'intérêt, les références, les méthodes bien au-delà de notre propre cercle géographique et culturel, la transmission de connaissances précises pourrait encourager le dialogue et contribuer à distinguer croyances et savoirs étayés. C'est pourquoi à SOLEIL, nous avons considéré que l'éducation non formelle prodiguée in situ, en complément des enseignements scolaires, pouvait également jouer un rôle pour contribuer à intéresser le public aux enjeux et aux choix scientifiques et technologiques.

C'est le travail de médiation qu'effectuent, au-delà des synchrotrons euxmêmes, de plus en plus de laboratoires et d'instituts. Ce mouvement, initié sous le label de "diffusion de la culture scientifique et technique", a pris un essor considérable en France au début des années 1980 et a, depuis, suscité des initiatives remarquables, évidemment en Île-de-France où le Palais de la découverte avait ouvert la voie et où la Cité des sciences a exploré d'autres dispositifs. Ce foisonnement s'exprime aussi en région. Des CCSTI, inspirés de la Casemate de Grenoble, ont ainsi peu à peu maillé le territoire national, de Bordeaux à Montbéliard, de Marseille à Nantes, sans oublier nos collègues d'Outre-Mer ${ }^{(2)}$. De nombreuses associations ont également vu le jour ou accru leur action, animées par des professionnels d'une discipline ou d'une démarche.

L'implication de SOLEIL dans cette dynamique a fait partie, dès 2001, de la vie du projet. L'objectif était de partager des connaissances disponibles dans un synchrotron avec quiconque en émettrait le souhait. Nous voulions évoluer ensemble dans la façon d'aborder et de discuter les sujets d'expertise d'un synchrotron. Comme il fallait bien commencer, " faire la première passe " comme on dit dans les sports d'équipe, SOLEIL a choisi d'engager le dialogue en s'appuyant sur des approches expérimentales concrètes et actives. Le Rectorat de Versailles nous y a beaucoup aidés dès le début en mettant à disposition à SOLEIL, deux jours par semaine, une enseignante qui pouvait se consacrer à tisser du lien avec ses collègues et les élèves de tous niveaux ${ }^{(3)}$.

Évidemment, la rhétorique de la transmission des connaissances et des compétences ne nous est pas étrangère et, modestement, nous la prenons en compte dans les pratiques que nous proposons, en particulier au monde enseignant. Cependant, si les synchrotrons sont des lieux transdisciplinaires - comme en témoignent les articles de ce dossier-, ce sont surtout des lieux d'accroissement des connaissances, des terrains expérimentaux où les meilleures équipes scientifiques du monde viennent explorer des champs théoriques et prolonger des démarches de recherche sur des sujets de science fondamentale majeurs. Les 


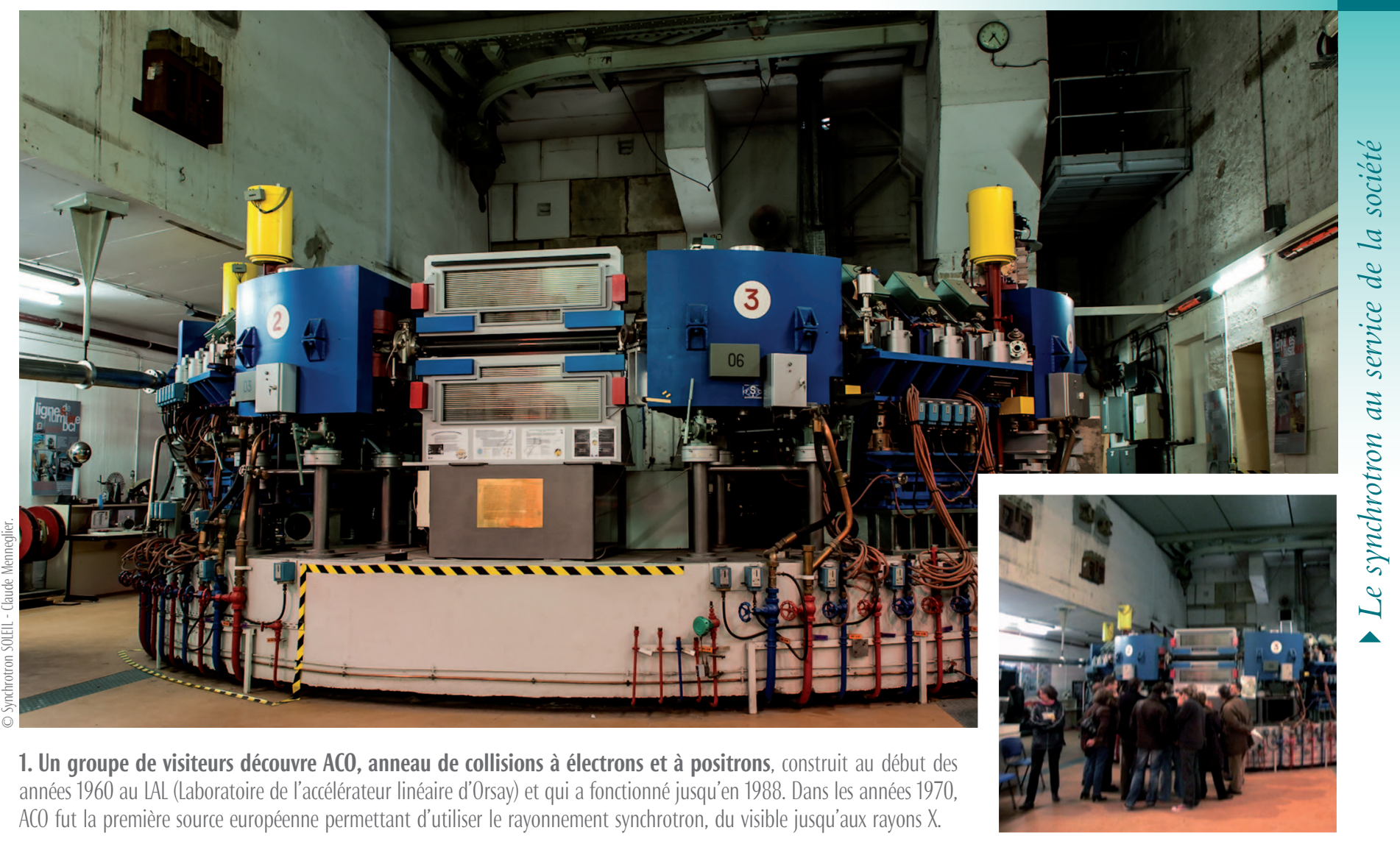

connaissances ne sont pas toujours immédiatement utiles, mais comprendre le plus simple des phénomènes reste une grande richesse.

Dès lors, la transmission des savoirs acquis jour après jour dans ces très grands instruments garde une valeur intrinsèque. L'effort que nous déployons pour imaginer comment les mettre en perspective avec les programmes scolaires nous permet par ailleurs de proposer des stages aux enseignants, au fur et à mesure que de nouvelles thématiques enrichissent les programmes. C'est le cas, par exemple, cette année, de la spectroscopie/microscopie infrarouge, qui n'était pas enseignée au lycée et dont les techniques ont considérablement évolué depuis quinze ans. Le montage de ces formations sur mesure, en collaboration avec les enseignants et les inspecteurs eux-mêmes, est aussi l'occasion de questionner ce qui pour nous, équipe de médiation scientifique, paraît "simple». La préparation de ces stages permet également aux équipes scientifiques d'accroître leur aisance pour communiquer avec leurs pairs ou avec des communautés académiques ou industrielles qui, ignorantes des techniques synchrotrons, pourraient pourtant en bénéficier dans leurs recherches.

La transmission des connaissances est, ici, toujours à double sens. Et comment pourrait-il en être autrement quand nos équipes scientifiques, quelles que soient leurs spécialités, accueillent chaque semaine des utilisateurs issus de toutes les disciplines : matériaux, biologie, chimie, environnement, géo et astrophysique, art, archéologie..

\section{S’appuyer sur la démarche de recherche}

Un synchrotron tourne en continu toute l'année. Quand tous les créneaux horaires sur toutes les lignes (on les appelle des shifts et ils durent huit heures) sont occupés, les utilisateurs doivent attendre le semestre suivant pour candidater. De la même façon, SOLEIL est ouvert au public tous les jours toute l'année, depuis bientôt dix ans, et une équipe de médiation scientifique de deux personnes dédiées à cette activité, assure les visites. Les visites durent en général entre 2 h 30 et $4 \mathrm{~h}$, et mobilisent une personne pour quinze visiteurs (une pour sept lorsqu'il s'agit de scolaires). Il n'est pas rare que nous soyons obligés de surseoir de plusieurs semaines à la programmation d'une rencontre. Les délais d'attente ne sont évidemment pas comparables avec ceux d'une installation unique comme le CERN. L'accès quotidien du public aux centres de recherche est encore rare et SOLEIL ne peut, dans les conditions actuelles, recevoir plus de 5000 visiteurs par an. Les visites se déroulent évidemment alors que les lignes de lumière fonctionnent. Les visiteurs circulent au même moment et dans les mêmes locaux que les équipes scientifiques.

La visite est donc au cœur de nos dispositifs de médiation. Elle peut être couplée à une visite de Sciences $\mathrm{ACO}^{(4)}$, un accélérateur pionnier du domaine (fig. 1), transformé en musée par une poignée de passionnés qui en assurent l'animation, et dont SOLEIL assure la direction. Cette ouverture sur l'histoire des sciences et des techniques est une autre ligne directrice de notre action. Elle permet d'insister sur la dimension collective de la recherche, sur l'importance de toutes les compétences, aussi surprenantes puissent-elles paraître a priori. Elle permet aussi de raconter les parcours professionnels des femmes et des hommes, des parcours pas toujours linéaires du bon élève en classe au bon chercheur en labo même si évidemment, l'excellence n'est pas un défaut. La complémentarité entre un lieu comme SOLEIL, à la pointe de la technologie, et un lieu comme Sciences ACO qui le fut en son temps, permet aussi de relativiser le traditionnel récit de la Science et la perception erronée que peuvent en avoir ceux qui s'en sentent spectateurs. 


\section{L'offre pédagogique de SOLEIL}

SOLEIL a mis en place un dispositif de communication complet (site web, actualités scientifiques relayées auprès de la presse, documents, événements). Ce dispositif assez classique permet de faire connaître le centre et ses réalisations à différents publics a priori concernés ou a minima intéressés par ses activités.

L'enjeu de la médiation est tout autre : il consiste littéralement à rendre perméables les frontières du laboratoire pour que le public puisse s'y sentir attendu. SOLEIL se visite donc tous les jours de la semaine, ainsi que quelques jours par an en soirée ou le week-end. Pour préparer une visite, il suffit de nous contacter par e-mail (visites@synchrotron-soleil.fr). Les visites sont gratuites et différents programmes sont proposés en fonction du niveau de familiarité avec les thématiques scientifiques ou des centres d'intérêts des groupes. Pour préparer ou prolonger la visite, SOLEIL a développé une offre éditoriale complète : huit kits pédagogiques (fig. E1), des brochures didactiques thématiques, des films et animations repris sur une téléweb (dailymotion et youtube)... L'ensemble est soit téléchargeable gratuitement sur le site web de SOLEIL (www.synchrotron-soleil, rubrique «documentation»), soit disponible sur commande via l'adresse webcom@synchrotron-soleil.fr .

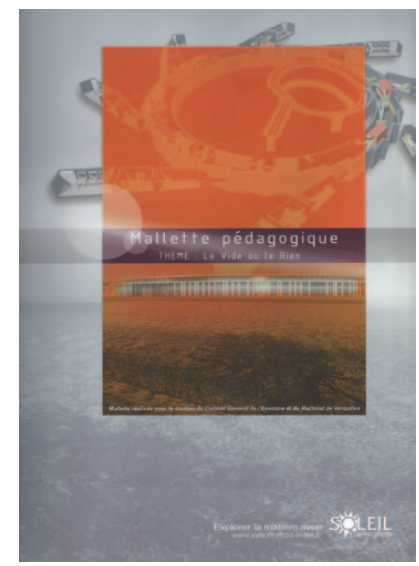

SOLEIL est également partenaire de la revue DocSciences (www.docsciences.fr), et apporte son soutien à de nombreux dispositifs pédagogiques : ASTEP, classes a-pac, TPE, TIPE, concours Quintesciences, C'génial, Les Olympiades, E2phy... Enfin, SOLEIL produit des expositions en prêt gratuit (panneaux souples de format A1 dans une valise de transport) et des ateliers pédagogiques itinérants (fig. E2).

E1. Mallette pédagogique « Le Vide ou le Rien ». Sont également disponibles: "Optique ", "Lumière synchrotron", "Sciences de l'univers", "Sciences de la vie et de la Terre ", « Le magnétisme "...

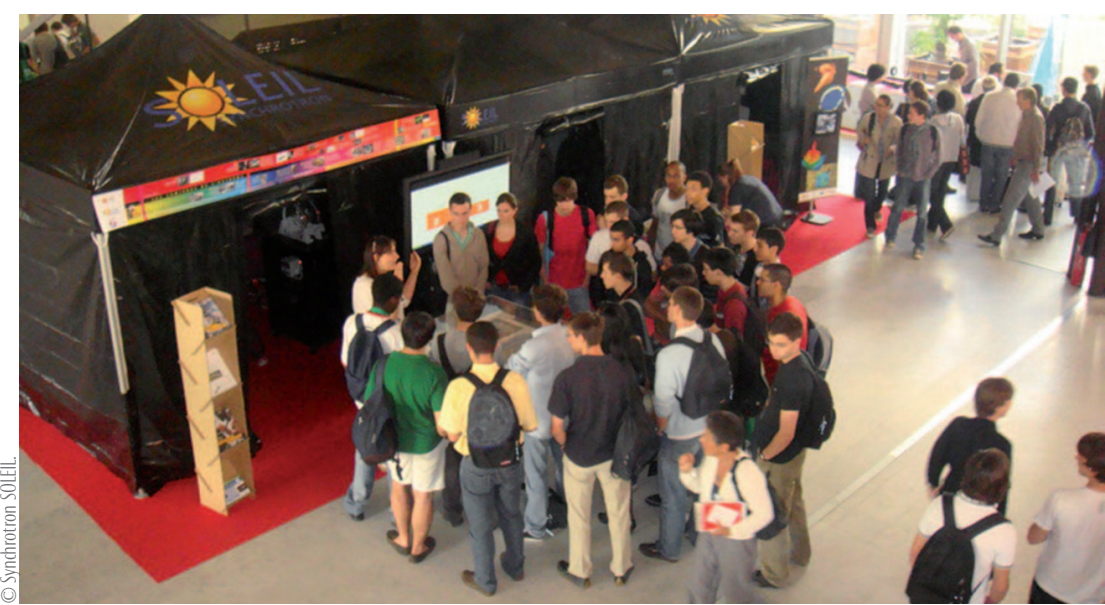

E2. Animation pédagogique hors les murs, lors de l’année du laser (2010).

(1) CCSTI : Centre de culture scientifique, technique et industrielle.

(2) SOLEIL est plus particulièrement partenaire - à leur demande - des CCSTI d'Orléans Centre Sciences et de la Réunion. L'ESRF, quant à lui, collabore étroitement avec la Casemate de Grenoble.

(3) Chantal Dhez, enseignante de physique à Verrières-le-Buisson, a collaboré avec SOLEIL de 2002 à 2004. Valérie Péduzy, enseignante de physique-chimie au lycée de la Vallée de Chevreuse à Gif-surYvette, nous a rejoint à SOLEIL ensuite, et poursuit son travail à nos côtés.

(4) ACO : Anneau de collision d'Orsay. Pour en savoir plus ou réserver une visite : www.sciencesaco.fr (5) HELIOS, une ligne de lumière dédiée aux apprentis chercheurs de tout âge et aux étudiants motivés (ouverture prévue en 2015 ; d'ici là, programme distribué sur les lignes actuelles de SOLEIL).

(6) Un Fab lab (contraction de l'anglais "Fabrication laboratory") désigne littéralement un atelier composé de machines-outils pilotées par ordinateur, pouvant fabriquer ou modifier rapidement des objets.

\section{\>}

En bons physiciens, professionnels ou autodidactes, s'il est un paramètre dont nous savons qu'il modifie ce que l'on voit, c'est bien le fait de le regarder. C'est pourquoi, dans notre démarche, ce que le public nous dit, nous renvoie, n'est jamais négligé. En retour, nous avons aussi une exigence : le respect mutuel dans le dialogue, car un scientifique est aussi un citoyen responsable qui s'interroge.

\section{S'appuyer sur des partenariats pour innover dans nos pratiques}

Les pratiques en médiation scientifique et technique sont, elles aussi, en perpétuel questionnement. Elles évoluent très vite : les expositions ont gagné en interactivité ; les animations sont de plus en plus courtes, elles sont déclinées sur une multitude de supports numériques et leurs concepteurs cherchent à étonner pour susciter la curiosité. Notre vocation n'est pas de surenchérir sur des techniques déjà très sophistiquées.

À SOLEIL, nous plaçons surtout nos efforts pour permettre au public de s'approprier le plus directement possible la réalité des problématiques et des métiers scientifiques. C'est ainsi que nous avons développé deux nouveaux dispositifs intitulés SOLEIL pursuit (fig. 2) et HELIOS $^{(5)}$, qui semblent correspondre aux attentes des enseignants et aussi d'un public de jeunes adultes, très difficile à atteindre, car il n'est plus forcément scolarisé. Ses centres d'intérêt se placent alors dans d'autres champs que l'acquisition de connaissances scientifiques (voir l'encadré).

Par ailleurs SOLEIL, aux côtés de l'Université Paris-Sud et d'autres partenaires locaux, participe au développement de deux projets de médiation singuliers. Le premier s'appelle la Maison d'Initiation et de Sensibilisation aux Sciences (MISS). Il s'agit d'un lieu dédié au questionnement et à l'expérimentation par le biais, notamment, d'ateliers scientifiques pour les scolaires. L'objectif est d'éveiller au plus tôt leur intérêt pour les sciences expérimentales et de développer leur démarche scientifique. Cette MISS, dont l'investissement est assuré par la Région Île-de-France, accueillera les cycles 3 (CE2, CM1, CM2) et les collèges franciliens pour des parcours scientifiques en lien avec les domaines d'expertises de l'Université Paris-Sud et des 


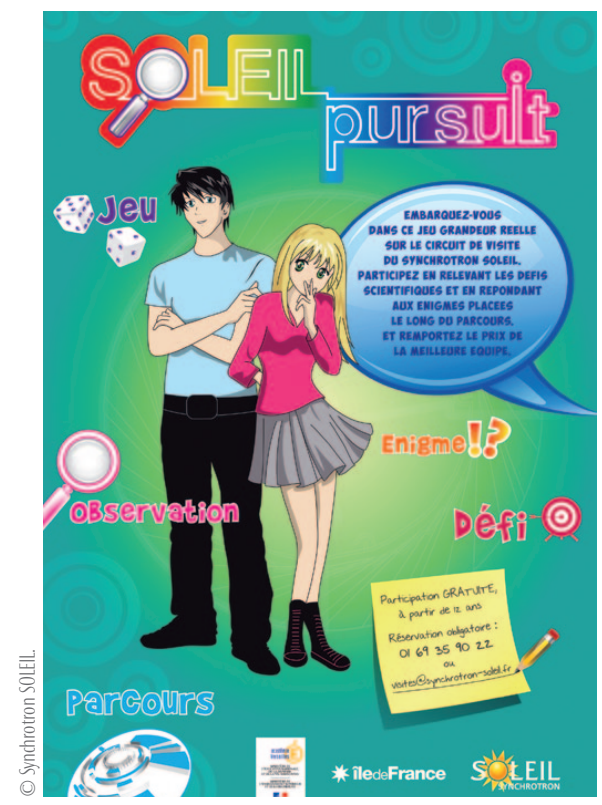

2. SOLEIL pursuit, un jeu de pistes dans le synchrotron, une alternative très interactive aux visites plus classiques.

autres établissements et associations de la future Université Paris-Saclay. L'ouverture aux classes est prévue à la rentrée 2014, après une phase de conversion et d'aménagement des locaux, situés sur le campus de l'université. À terme, la MISS pourrait accueillir des classes et des enseignants en résidence sur plusieurs jours.

La MISS est inscrite dans la dynamique d'une plate-forme en cours de création intitulée "La Diagonale Paris-Saclay", qui fait partie du projet d'initiative d'excellence (Idex) Paris-Saclay et a pour ambition de doter le futur campus d'un grand centre de dialogue science \& société et d'animation scientifique. Elle traduit la volonté d'une interaction directe entre tous les acteurs de la société : population locale, étudiants, artistes, scientifiques, industriels, acteurs sociaux et politiques... Pour ce faire et en parallèle du volet destiné spécifiquement au monde enseignant, elle développera également un axe "Arts et Sciences" articulé autour d'actions de recherche, de création, d'enseignement : accueil en résidence d'artistes, spectacles, création d'œuvres, Fab $l a b^{(6)} \ldots$

Le troisième volet prioritaire de cet ambitieux programme concerne la valorisation du patrimoine scientifique et technique. Il s'agit, plus particulièrement, de la mise au point d'une démarche et d'outils pour la valorisation, la préservation et la sensibilisation à la mémoire des objets, des lieux et des pensées. 